\title{
Erhalten bald auch Pauschalerstattungen eine Zeitvorgabe?
}

- Die Leistungen der aktuellen Version des Einheitlichen Bewertungsmaßstabes (EBM) sind betriebswirtschaftlich kalkuliert. Ihre Bewertung errechnet sich aus einem Kostenanteil (TL) und einem Arztlohn (AL). Die Kalkulationsbasis ist dabei 5,11 Cent. Da diese Vergütungshöhe zurzeit (immer) noch durch gesetzliche Rahmenbedingungen auf rund 3,5 Cent reduziert ist, wird im Anhang 3 des EBM zwischen einer Kalkulationszeit und einer Prüfzeit unterschieden.

Die Prüfzeit ist dabei in der Regel geringer angesetzt als die Kalkulationszeit, ist aber Grundlage für die - ebenfalls gesetzlich vorgeschriebenen - Plausibilitätsprüfungen nach Zeitvorgaben. Einige Leistungen, die nicht in dieser Form kalkuliert wurden, wie z.B. die Kostenpauschalen des Abschnitts 40 des EBM, wurden bisher von dieser Form der Wirtschaftlich- keitsprüfung ausgeschlossen. Das soll sich nach dem Willen der Kassen an einigen Stellen ändern. Geplant ist, die neu geschaffenen Pauschalvergütungen für die Verordnung der Palliativversorgung (Nr. 40860 EBM Erstverordnung, Nr. 40862 EBM Folgeverordnung) und die Anträge zum Zweitmeinungsverfahren bei bestimmten Arzneimittelbehandlungen (Nr. 40865 EBM Erstantrag, Nr. 40866 EBM Folgeanträge) einer solchen Plausibilitätsprüfung nach Zeitvorgaben zu unterziehen.

\section{MMW Kommentar}

Wenn es um den Aufbau bürokratischer Monster-meist ohne entsprechende finanzielle Gegenleistung - geht, waren die Kassen schon immer schnell bei der Hand. Die jetzt geplanten Regelungen stellen allerdings alles in den Schatten, was auf dieser Ebene bisher passiert ist. Eine Palliativbehandlung stellt bekanntlich eine Maßnahme dar, die bei einem Patienten notwendig wird, der nicht mehr lange zu leben hat und dem die verbleibende Zeit einigermaßen angenehm und vor allem schmerzfrei gestaltet werden soll. Kommt es tatsächlich zu einer solchen Einbeziehung der Verordnung einer Palliativbehandlung in das Zeitraster, könnte die unmenschliche Situation entstehen, dass ein Hausarzt gegen Ende des Quartals eine solche Verordnung nicht mehr ausstellen kann, weil sein Zeitkontingent erschöpft ist. Alternativ könnte er allenfalls die Leistung erbringen, aber nicht abrechnen. Es drängt sich deshalb der Verdacht auf, dass dies möglicherweise das Ziel der Maßnahme ist.

\section{Tabelle 1}

\section{Werden auch diese Leistungen bald nach Zeitvorgaben geprüft?}

\section{GOP Kurzlegende}

40860 Kostenpauschale zur Erstattung des besonderen Aufwandes im Rahmen der Erstverordnung der spezialisierten ambulanten Palliativversorgung gemäß der Richtlinie des Gemeinsamen Bundesausschusses nach $\S 37$ b SGB V

40862 Kostenpauschale zur Erstattung des besonderen Aufwandes im Rahmen der Folgeverordnung zur Fortführung der spezialisierten ambulanten Palliativversorgung gemäß der Richtlinie des Gemeinsamen Bundesausschusses nach $§ 37$ b SGB V

40865 Erstantrag besondere Arzneimitteltherapie zur Behandlung der pulmonal-arteriellen Hypertonie unter Verwendung des Musters 71 gemäß Anlage XI zum Abschnitt N der Arzneimittel-Richtlinie des Gemeinsamen Bundesausschusses nach $§ 92$ Abs. 1 Satz 2 Nr. 6 SGB V

40866 Erster oder zweiter Folgeantrag besondere Arzneimitteltherapie zur Behandlung der pulmonalarteriellen Hypertonie unter Verwendung des Musters 71A gemäß Anlage XI zum Abschnitt N der ArzneimittelRichtlinie des Gemeinsamen Bundesausschusses nach § 92 Abs. 1 Satz 2 Nr. 6 SGB V
Kalkulationszeit Prüfzeit in Eignung der in Minuten

KA Minuten Prüfzeit

Tages- und Quartalsprofil

KA

11

Tages- und Quartalsprofil

KA 13

Tages- und Quartalsprofil

KA 7

Tages- und Quartalsprofil 